$\begin{array}{cl}\begin{array}{c}\text { Revue } \\ \text { de } / \text { histoire } \\ \text { des religions }\end{array} & \text { Revue de l'histoire des religions } \\ & \begin{array}{l}1 \mid 2014 \\ \text { Varia }\end{array}\end{array}$

\title{
Mgr Agostino Casaroli, un habile « tisseur de dialogues européens » (1963-1975)
}

Monsignor Agostino Casaroli, a skilful "weaver of European dialogues"

(1963-1975)

Marco Lavopa

\section{OpenEdition}

\section{Journals}

Édition électronique

URL : http://journals.openedition.org/rhr/8196

DOI : $10.4000 /$ rhr.8196

ISSN : 2105-2573

Éditeur

Armand Colin

Édition imprimée

Date de publication : 1 mars 2014

Pagination : 101-115

ISBN : 978-2200929107

ISSN : 0035-1423

\section{Référence électronique}

Marco Lavopa, « Mgr Agostino Casaroli, un habile «tisseur de dialogues européens » (1963-1975)»,

Revue de l'histoire des religions [En ligne], 1 | 2014, mis en ligne le 01 mars 2017, consulté le 05 mai

2019. URL : http://journals.openedition.org/rhr/8196 ; DOI : 10.4000/rhr.8196 


\title{
Mgr Agostino Casaroli, un habile «tisseur de dialogues européens » (1963-1975)
}

\begin{abstract}
À travers l'analyse de l'œuvre diplomatique d'Agostino Casaroli, on se propose d'étudier les raisons historiques qui ont mené le Saint-Siège (sous le pontificat de Paul VI) à entreprendre des négociations avec les États socialistes de l'Est européen, et à choisir la «politique du dialogue». Mgr Casaroli est le représentant d'une diplomatie vaticane qui se trouve au centre de la scène européenne et internationale pendant les années de la "Guerre froide», au niveau tant bilatéral que multilatéral (avec la participation de Mgr Casaroli lui-même - comme délégué du SaintSiège - à la Conférence d'Helsinki de 1975). L'enjeu principal était de permettre l'existence de l'Église catholique dans le monde communiste.
\end{abstract}

\section{Monsignor Agostino Casaroli, a skilful "weaver of European dialogues" (1963-1975)}

The present paper aims to analyze the diplomatic work of Agostino Casaroli in order study the historical reasons that led the Holy See (under the pontificate of Paul VI) to undertake negotiations with the socialist states of Eastern Europe and choose the "politics of dialogue". Monsignor Casaroli is the representative of a Vatican diplomacy that lies at the center of the European and international scene during the years of the Cold War, both bilaterally and multilaterally (with the participation of Bishop Casaroli himself - as a delegate of the Holy See - in the Helsinki Conference of 1975). The main purpose was to allow the existence of the Catholic Church in the communist world. 
L'œuvre principale de Mgr Agostino Casaroli: «l'Ostpolitik vaticane», ainsi caractérisée par l'historiographie contemporaine', s'accomplit durant une période de changements rapides en Europe et dans le monde, notamment en Europe de l'Est, et plus particulièrement entre 1963 et 1975 . Un long et habile travail de «tisseur de dialogues» et de «constructeur de la paix», voilà l'aspect essentiel de l'action de cet infatigable diplomate - il a accompagné le ministère de cinq pontifes: Pie XII, Jean XXIII, Paul VI, Jean Paul I ${ }^{\text {er }}$, Jean Paul II - au service du Saint-Siège dans le monde (et pas seulement communiste) $)^{2}$.

\section{Un HOMME FORMÉ AU SEIN dU VATICAN}

Agostino Casaroli naît à Castel S. Giovanni, diocèse de PiacenzaBobbio, le 24 novembre 1914. À l'âge de 11 ans, il entre au séminaire de Bedonia (Parme) pour accomplir ses études et devenir prêtre (le 27 mai 1937). Casaroli assiste à des cours préparatoires à la diplomatie vaticane auprès de l'Académie pontificale ecclésiastique, et en novembre 1939 il obtient le titre de docteur en Droit canonique à l'université pontificale du Latran. Exempté du service diplomatique dans les différents sièges à l'étranger pour des raisons de santé, Casaroli entre à la Secrétairerie d'État à 26 ans, en 1940 (sous le pontificat de Pie XII), comme archiviste.

En 1961 le pape Jean XXIII (élu en 1958) le nomme soussecrétaire dans la $2^{\text {e }}$ Section de la Secrétairerie d'État, la Sacrée Congrégation pour les affaires ecclésiastiques extraordinaires qui après la réforme de 1967 prendra le nom de Conseil pour les Affaires publiques de l'Église (et après la réforme de 1988, Section pour les Relations avec les États). Casaroli a donc effectué toute sa carrière au sein de la Secrétairerie d'État (dont il deviendra le titulaire sous

1. Voir Agostino Giovagnoli, "Ostpolitik: un bilancio storiografico», in Achille Silvestrini éd., L'Ostpolitik di Agostino Casaroli 1963-1989, actes de la table ronde organisée à l'occasion du dixième anniversaire de la mort du cardinal A. Casaroli, Bedonia (Parme) 10 juin 2008, Bologne, EDB, 2009, p. 103-131.

2. Sur ces questions, voir Giovanni Barberini, L'Ostpolitik della Santa Sede: un dialogo lungo e faticoso, Bologne, Il Mulino, 2007. 
Jean Paul II) ${ }^{3}$, sans aucune formation au service diplomatique au-dehors des murs du Vatican, mais graduellement enrichie par des voyages et des rencontres inédits pour un ecclésiastique en ces périodes de l'histoire. En outre il faut rappeler que la $2^{\mathrm{e}}$ Section de la Secrétairerie d'État fut fondée pour faire face aux extraordinaires bouleversements de la Révolution française et aux nouvelles questions ecclésiastiques qu'elle posait. En effet, la Sacra Congregatio pro negotiis ecclesiasticis extraordinariis développa ses propres activités à partir de la confrontation avec l'État français révolutionnaire puis avec le Consulat et l'Empire napoléoniens. Ce sont ces bouleversements qui obligèrent le Saint-Siège à créer un nouvel instrument (la Congrégation pour les affaires ecclésiastiques du royaume des Gaules, en 1793, devenue la Sacrée Congrégation pour les affaires ecclésiastiques extraordinaires en 1814), qui fût apte à traiter les affaires avec des hommes et des institutions se mettant, pour la première fois dans l'histoire de l'Église, en dehors de l'horizon «naturel» de la catholicité. Le Saint-Siège se vit contraint de reconnaître l'existence de l'État-nation, à traiter avec les États sortis des révolutions latino-américaines, l'État laïque, l'État autoritaire, fasciste ou nazi, l'État communiste, etc. À partir de la Révolution française, le Saint-Siège comprit la nécessité de composer avec des autorités étrangères voire hostiles au monde chrétien, ou avec des hommes politiques qui, même lorsqu'ils étaient de confession catholique, exerçaient leur action sans référence religieuse, en vertu du principe de laïcité. De cette tradition ecclésiastique et de cette école diplomatique sont issus les secrétaires d'État du XIX ${ }^{\mathrm{e}}$ et du $\mathrm{XX}^{\mathrm{e}}$ siècle.

3. «Il devient, par décision du pape Paul VI, en 1967, secrétaire de la même Sacrée Congrégation. Le 16 juillet 1967 il est consacré évêque par le pape Paul VI, titulaire du siège archiépiscopal de Carthage. Le 28 avril 1979 il est nommé secrétaire d'État du Vatican et préfet du Conseil pour les affaires publiques de l'Église par le pape Jean Paul II, et devient cardinal le 30 juin de la même année.» Carlo Felice Casula, «Agostino Casaroli : nota biografica», in Agostino Casaroli, Il martirio della pazienza: La Santa Sede e paesi comunisti (1963-1989), C.F. Casula et Giovanni Maria Vian éd., Turin, Einaudi, 2000, p. XXXI-XXXV. 


\section{L'HOMMe de L'Est aU VATICAN}

Les régimes communistes d'Europe de l'Est pouvaient être considérés comme l'aboutissement de l'époque des révolutions, un produit de la sécularisation de l'État, avec une nouvelle position très claire par rapport à la religion: l'athéisme d'État. Le SaintSiège avait déjà élaboré les instruments diplomatiques et juridiques pour traiter avec les États laïques et avec les États autoritaires, mais la négociation avec un État athée apparaissait comme une véritable absurdité pour beaucoup de hauts représentants de l'Église catholique elle-même. Mgr Casaroli sut au contraire dégager la voie pour une action diplomatique concrète. À travers ses notes au souverain pontife et au secrétaire d'État du Vatican sur ses rencontres et sur les résultats auxquels il parvenait, il se révéla bon connaisseur des pays communistes et fin analyste de ce qui se passait au-delà du «rideau de fer $»^{4}$. Il commençait toujours par l'interprétation exacte des règles en vigueur dans chaque État, la connaissance du contexte dans lequel s'inscrivaient ces normes, et après avoir fait les critiques de principe il en tirait les conséquences pour de possibles relations futures.

Ces pages très importantes de l'activité politique internationale du Saint-Siège au cours du second après-guerre conduisirent à sa participation à la Conférence pour la sécurité et la coopération en Europe (CSCE) dite conférence d'Helsinki. Il faut rappeler que, depuis le Congrès de Vienne en 1815 , c'était la première fois que le Saint-Siège participait pleno jure à un symposium politique multilatéral. Il s'agissait à Helsinki non pas de résoudre des conflits territoriaux, mais d'aborder en commun des affaires politiques, relatives à la sécurité, à la coopération et à la stabilité entre les États du continent européen.

Pour cette raison, la conférence d'Helsinki doit être évaluée non comme une initiative occidentale naïve, mais bien comme une tentative des deux parties, l'Est et l'Ouest, de dépasser la division de l'Europe en blocs et d'établir ensemble, dans les limites du possible, une base politique et culturelle. Casaroli fut l'un des grands protagonistes et un défenseur de la CSCE: il affirmait que la

4. Voir La politica del dialogo. Le carte Casaroli sull'Ostpolitik vaticana, G. Barberini (éd.), Bologne, Il Mulino, 2008. 
conférence d'Helsinki pouvait être une contribution historique que le Saint-Siège donnait au monde, dans la perspective de la création d'un même cadre à l'Est et à l'Ouest de l'Europe.

Soutenu par un fort sens du réalisme, Mgr Casaroli se montra dans cette longue histoire des dialogues impossibles l'homme des grands desseins. Sa conviction était que dans les pays communistes, après le temps désolant des incarcérations et des déportations qui avaient dépeuplé les diocèses, il était nécessaire de rétablir une hiérarchie d'évêques dignes et moralement fidèles à l'Église de Rome, tout en étant reconnus par les gouvernements en place, afin de pourvoir à l'encadrement pastoral de la majorité des catholiques: l'exemple de la Tchécoslovaquie montrait que les formations ecclésiales clandestines ne permettaient pas d'assurer la vie religieuse des populations, car elles étaient inévitablement élitaires. Le Saint-Siège ne voulait pas se contenter des simples nominations épiscopales. En effet, les évêques comme le clergé devaient avoir une possibilité réelle d'exercer leur ministère, donc de rétablir les communications normales et essentielles avec le Saint-Siège.

Toutefois, les concessions qu'il fallait faire dès lors que ces questions étaient considérées comme prioritaires, étaient jugées par d'autres «réductrices et inefficaces». Le secrétaire d'État luimême, le cardinal Villot, affirma avec une certaine ironie que le modus vivendi était plutôt un modus non moriendi: une manière de ne pas mourir, plutôt qu'une manière de vivre. Les opposants à la diplomatie de Casaroli, malgré la nouveauté du concile Vatican II (1962-1965) et peut-être aussi parfois contre elle, se recrutaient parmi les catholiques de l'Est eux-mêmes et dans une fraction de la Curie romaine, fortement anticommuniste: leur point de vue était que l'Église ne peut pas, et ne doit pas, pactiser avec l'ennemi ${ }^{5}$. Cependant Mgr Casaroli réussit à imposer l'idée que la survie de l’Église dans les pays communistes passait par une reconnaissance légale et donc la possibilité d'une action diplomatique. La diplomatie vaticane devint dans ce contexte un outil stratégique, permettant au gouvernement central de l'Église romaine de connaître et

5. Sergio Trasatti, La croce e la stella: la chiesa e $i$ regimi comunisti in Europa dal 1917 a oggi, Milan, Mondadori, 1993; A. Riccardi, «Antisovietismo e Ostpolitik della Santa Sede da Benedetto XV a Paolo VI», in Alberto Melloni e Maurilio Guasco éd., Un diplomatico vaticano fra dopoguerra e Ostpolitik. Mons. Mario Cagna (1911-1986), Bologne, Il Mulino, 2003, p. 123-173. 
d'accueillir les «nouveaux signes des temps». L'Église comprit en cette période la nécessité d'entreprendre de nouvelles politiques pour jouer un rôle dans ce monde moderne de plus en plus assujetti à des processus de sécularisation.

\section{La nature de l'Ostpolitik de Mgr Casaroli}

Cette action diplomatique aboutit à l'acceptation par les régimes marxistes-léninistes des positions exprimées par Jean XXIII dans l'encyclique Pacem in Terris ${ }^{6}$ et plus généralement celles du concile Vatican II (en particulier la constitution pastorale Gaudium et spes ${ }^{7}$, 1965).

Sur le plan de la méthode, la fin du concile ouvrait un espace à la «politique du dialogue» théorisée par Paul VI dans l'encyclique Ecclesiam suam ${ }^{8}$, où Mgr Casaroli investit une nouvelle génération de diplomates, capable d'apprendre un nouveau style (inauguré par Casaroli lui-même et non dispensé à l'Académie pontificale ecclésiastique) et d'opérer sur le délicat terrain de l'Ostpolitik. Il a ainsi formé, au sein du Conseil pour les affaires publiques de l'Église qu'il présidait, «Achille Silvestrini, Pio Laghi, Angelo Sodano, Justin Rigali, Giovanni Cheli, Luigi Bongianino, Mario Cagna (représentant pontifical en Yougoslavie), Luigi Poggi (nommé en 1973 nonce spécial avec compétence en Pologne, Hongrie, Tchécoslovaquie, Roumanie, Bulgarie), Francesco Colasuonno (pro-nonce en Yougoslavie depuis 1985, en Pologne depuis 1986 et en URSS depuis 1990) et Corrado Bafile (nonce en Allemagne avec siège en RFA)»9.

Mgr Poggi, collaborateur de Mgr Casaroli, rappela pendant une réunion avec les représentants du gouvernement tchécoslovaque

6. Jean XXIII, lettre encyclique Pacem in terris, www.vatican.va/holy_ father/john_xxiii/encyclicals/documents/hf_j-xxiii_enc_11041963_pacem_ fr.html

7. Constitution pastorale sur l'Église dans le monde de ce temps Gaudium et spes, www.vatican.va/archive/hist_councils/ii_vatican_council/documents/vat-ii_ const_19651207_gaudium-et-spes_fr.html

8. Paul VI, lettre encyclique Ecclesiam suam, www.vatican.va/holy_father/ paul_vi/encyclicals/documents/hf_p-vi_enc_06081964_ecclesiam_fr.html

9. A. Melloni, «L'Ostpolitik e i suoi uomini», in A. Melloni et M. Guasco éd., Un diplomatico vaticano fra dopoguerra e Ostpolitik. Mons. Mario Cagna (19111986), Bologne, Il Mulino, 2003, p. 241-242. 
que «la délégation a été présidée toujours par Mgr Casaroli [...]. Il a été choisi par le pape Paul VI pour incarner sa bienveillante et clairvoyante attitude envers la Tchécoslovaquie et envers les autres pays socialistes. $\gg^{10}$ Toute la ligne d'action de Casaroli sera soutenue par les papes Jean XXIII et Paul VI, et encouragée par le nouveau contexte international. Cette reconsidération du monde moderne de la part du Vatican poussa l'Union soviétique à rechercher de façon presque continue des contacts avec la Secrétairerie d'État et à favoriser la participation du Saint-Siège à la conférence d'Helsinki - en dépit du scepticisme de certains États européens de l'Ouest et de quelques membres de la Curie romaine elle-même - et à insister sur l'adhésion du Saint-Siège au Traité sur la non-prolifération des armes nucléaires (TNP).

Le gouvernement soviétique se montra fort sensible à la présence de Mgr Casaroli à Moscou (l'une des trois villes autorisées à recevoir les instruments de la ratification), où il déposa, le 25 février 1971, l'instrument d'adhésion du Vatican au TNP ${ }^{11}$. En cette circonstance il y eut des rencontres entre les représentants du Vatican et les autorités soviétiques dont le caractère fut purement politique, et où fut notamment abordé le sujet de la Conférence sur la sûreté et la coopération en Europe.

En mai 1969 le gouvernement finlandais avait officiellement présenté au Saint-Siège, à propos de la possibilité d'organiser cette conférence, un aide-mémoire qui fut envoyé en même temps à tous les gouvernements européens et à ceux des États-Unis et du Canada. La Finlande offrait d'accueillir la conférence prévue. «La réponse donnée par le Saint-Siège, après plusieurs sondages auprès des représentants des pays appartenants aux deux blocs, est contenue dans deux mémorandums de l'octobre suivant, envoyés aux États membres du Pacte de Varsovie et à la Finlande $»^{12}$.

10. Verbale della riunione con la delegazione cecoslovacca (16 gennaio 1980), a cura di Mons. Sainz Muñoz, in Archivio di Stato di Parma [désormais abrégé A.S.Pa.], Fondo Casaroli, b. 5 «Cecoslovacchia», f. 51, s.f. 3.

11. Dichiarazione addizionale allo strumento di adesione al trattato di non proliferazione delle armi nucleari «Rapp. N. 1050/71》 du 25 février 1971, in A.S.Pa., Fondo Casaroli, b. 2 «URSS», f. 4, s.f. 2. Sur le sujet, voir G. Barberini, «Mons. Casaroli nella politica internazionale», www.statoechiese.it/images/ stories/2009.7/barberini_mons.m.pdf, spéc. p. 4-9.

12. A. Casaroli, «La Santa Sede e l'Europa», conférence tenue le 20 janvier 1970 à l'ISPI (Institut d'études politiques internationales) de Milan, La Civiltà Cattolica, 19 février 1972, p. 379. 
En octobre 1969, le mémorandum du Conseil pour les affaires publiques de l'Église ${ }^{13}$, en réponse à la «Déclaration de Budapest» du mois de mars de la même année, suscita l'intérêt de la diplomatie internationale. Le 31 juillet 1970, après la visite du chancelier allemand Willy Brandt au Vatican, le Saint-Siège adressa aux gouvernements de tous les États européens ainsi qu'à ceux des États-Unis et du Canada un «Mémorandum sur la Conférence pour la sécurité en Europe $»^{14}$.

De plus, en ces mêmes années, la Secrétairerie d'État suivait avec beaucoup d'attention l'Ostpolitik du chancelier Willy Brandt, qu'elle considérait comme un instrument utile au soutien de sa propre ligne politique, particulièrement après la signature des traités entre la République fédérale d'Allemagne, l'Union soviétique et la Pologne, puis après la signature du traité fondamental entre les deux États allemands en 1972.

Toutefois, quand le Saint-Siège, avec Mgr Casaroli, commença de dialoguer directement avec la République démocratique allemande (on ne doit pas oublier l'avancée diplomatique qu'a représenté l'accord concernant les circonscriptions ecclésiastiques en RDA), il dut faire très attention aux susceptibilités et réserves des catholiques d'Allemagne fédérale (y compris les évêques) sur la question de la RDA. L'intérêt du Saint-Siège pour l'ouverture d'une table ronde avec l'Allemagne orientale s'expliquait par la nécessité pour la RDA d'entrer dans le jeu de la diplomatie internationale. Au cours du rendez-vous du 6 juillet 1973 à Helsinki entre Mgr Casaroli et le ministre des Affaires étrangères de la RDA, Otto Winzer, fut abordée la question de la création des diocèses dans la RDA et d'une conférence épiscopale de la RDA, requêtes devant lesquelles Casaroli se montra néanmoins très prudent ${ }^{15}$. Il s'agissait d'une

13. Le texte du mémorandum du Saint-Siège du 10 octobre 1969, recueilli dans les Politisches Archiv Auswärtigen Amts, Berlin [désormais abrégé PAAA], B 40 198 (BRD), les Politisches Archiv Auswärtigen Amts, Ministerium für Auswärtiges Angelegenheiten, Berlin-Est [désormais abrégé PAAA, Bestand MfAA], C 592/76 et C 157/77 (DDR), a été publié par Massimo Faggioli, «La Santa Sede e le due Germanie nel processo CSCE: dai documenti diplomatici della BDR e della DDR (1969-1974)», in A. Melloni éd., Il filo sottile. L'Ostpolitik vaticana di Agostino Casaroli, Bologne, Il Mulino, 2006, p. 179, note 18.

14. Sicherheit und Zusammenarbeit in Europa (KSZE), Hans Adolf Jacobsen, Wolfgang Mallmann et Christian Meier éd., doc. n. 61.

15. À propos de l'entrevue entre O. Winzer et Mgr Casaroli du 6 juillet 1973 à Helsinki, sur la question des diocèses, voir le rapport écrit par Herbert Plaschke, in 
nouvelle tentative pour offrir une solution juridique, dans l'un des pays de l'Europe de l'Est, antireligieux constitutionnellement, permettant la liberté de culte et l'organisation religieuse.

Le parcours était sûrement difficile, les résultats lents et insuffisants, les oppositions nombreuses y compris parmi les membres de la hiérarchie catholique; et cependant Mgr Casaroli fit la preuve que le Vatican pouvait obtenir par cette voie de nommer des évêques, communiquer avec eux librement, faire ouvrir les séminaires à nouveau, etc. Une analyse de la «section Europe occidentale» du ministère des Affaires étrangères (MfAA) de Berlin-Est confirmait l'intérêt du Saint-Siège pour améliorer ses relations avec les États socialistes: «actuellement le Vatican a entrepris une série d'initiatives extrêmement concentrées à l'égard des États socialistes (visite de Mgr Casaroli en Pologne, la solution du cas Mindszenty, une éventuelle visite de Casaroli à Cuba)» ${ }^{16}$. Les réflexions que Mgr Casaroli a consacrées aux États du socialisme réel dans les importants discours et conférences dédiés aux événements politiques et aux problèmes qui concernaient le continent européen après le Second Guerre mondiale, prononcés à Rome, à Milan, à Toronto, à New York, à Vienne, à Linz, à San Francisco et recueillis dans le volume Nella Chiesa per il mondo, peuvent être considérées comme un autre signe de l'évidente autorité qu'on lui reconnaissait sur la scène internationale.

\section{UNE ÉGLISE ADAPTÉE AU MONDE MODERNE :}

l'action de Mgr Casaroli à Helsinki

Ainsi Agostino Casaroli apparaît comme l'une des figures les plus intéressantes de l'histoire de l'Église catholique à l'époque

Dokumente zur Deutschlandpolitik, Herausgegeben vom Bundesministerium des Innern und vom Bundesarchiv, VI. Reihe/Band 3, Daniel Hofmann éd., Munich, R. Oldenbourg, 2002, p. 217-219.

16. Document du $1^{\text {er }}$ avril 1974, in PAAA, Bestand MfAA, C 160/77; d'après M. Faggioli, op. cit., p. 204, note 62. Sur le «cas Mindzenty» voir Gabriel Adriányi, Die Ostpolitik des Vatikans 1958-1978 gegenüber Ungarn. Der Fall Kardinal Mindszenty, Herne, Schäfer, 2003. Sur la visite de Mgr Casaroli à Cuba (1974), voir Riccardo Cannelli, «Il viaggio a Cuba di monsignor Casaroli», in L'America Latina fra Pio XII e Paolo VI. Il Cardinale Casaroli e le politiche vaticane in una Chiesa che cambia, A. Melloni et Silvia Scatena éd., Bologne, Il Mulino, 2006, p. 195-235. 
contemporaine. Au début du 1973, l'ambassadeur de RDA à Rome, Klaus Gysi, faisait observer que, après une période marquée par la volonté de Paul VI de décider personnellement à propos des questions de politique étrangère, «depuis quelque temps, le pape remet de plus en plus les décisions à Casaroli $»^{17}$. Sa grande capacité d'intervention en fit l'un des personnages-clés de ces années-là, celles de la guerre froide et du péril nucléaire. Selon ses propres paroles ces questions requéraient « courage et franchise, des qualités essentielles si nous voulons non pas rester des spectateurs et des victimes de l'Histoire mais, si nous en avons la possibilité, être de ceux qui déterminent cette Histoire $»^{18}$.

Pas moins important est son engagement en faveur de la paix, des droits de l'homme et du désarmement, à l'Est comme à l'Ouest: «Le Saint-Siège estime que les finalités du désarmement et de la détente qui inspirent le Traité correspondent à sa mission de paix, c'est-à-dire à la constante diligence avec laquelle il s'efforce de réaliser la sécurité, la mutuelle confiance, la coopération pacifique dans les relations entre les peuples $»^{19}$.

Casaroli avait le souci que l'Église ne se dérobe pas à sa mission dans le monde moderne, en cela offrant la diplomatie cohérente avec l'ecclésiologie du concile Vatican II, agissant conformément à l'encyclique Gaudium et spes, avec «joie et espoir». Sa maîtrise était évidente aussi dans sa capacité à concilier des intérêts spirituels, religieux et ecclésiaux, dans des négociations complexes, face à des gouvernements pourtant peu disposés à accorder des

17. Rapport de Klaus Gysi du 19 avril 1973, in PAAA, Bestand MfAA, C 160/77; d'après M. Faggioli, op. cit., p. 202, note 59.

18. «Die neue Stellung Europas», discours du 17 mars 1992 à l'université de Sienne, repris dans Wegbereiter zur Zeitenwende, Berlin, Duncker und Humblot Verlag, 1999, p. 105. Casaroli s'explique aussi sur l'Europe future et son rapport aux autres grandes puissances: «Cela concerne dans le concret, surtout, le rapport avec la seule superpuissance du monde, l'Amérique du Nord, qui pourrait être tentée, peut-être, dans l'un ou l'autre champ, d'exercer une sorte de "suprématie". Elle dispose de beaucoup de responsabilité, mais aussi de beaucoup de pouvoir et elle est instinctivement jalouse des tentatives d'autonomie, surtout celles qui pourraient être en conflit avec elle et qui pourraient transformer le nouvel ordre du monde en un "désordre mondial", éventuellement même en s'alliant avec d'autres puissances que le rêve de ce "nouvel ordre" n'intéresse pas (je pense particulièrement à la Chine, cette grande inconnue).»

19. Discorso di Mons. Casaroli per l'adesione della Santa Sede al Trattato di non-proliferazione nucleare del febbraio 1971, in A.S.Pa., Fondo Casaroli, b. 2 «URSS», f. 4, s.f. 3. 
privilèges à l'Église - sauf à les utiliser politiquement - et des pouvoirs politiques forts qui percevaient l'Église de Rome d'abord comme une puissance adverse. Dans un discours prononcé à New York, en 1973, au siège du Council on Foreign Relations, Mgr Casaroli traita directement le sujet du rapport entre le SaintSiège et l'Europe de l'Est, en fournissant des éléments-clés pour une juste appréciation de l'Ostpolitik vaticane: il le situait dans la problématique plus vaste des relations entre l'Église catholique et le monde moderne.

Le Conseil pour les affaires publiques de l'Église (dirigé par Mgr Casaroli avec l'aide de son collaborateur privilégié Mgr Achille Silvestrini) plaidait pour la présence du Saint-Siège à la conférence d'Helsinki. Selon Mgr Casaroli, la participation du Saint-Siège renforcerait sa position auprès des gouvernements socialistes et lui permettrait de protester publiquement contre l'absence de libertés religieuses dans les pays de l'Europe orientale. Une conférence européenne aiderait à dépasser la logique des blocs ${ }^{20}$.

Le 28 mars 1972 l'ambassadeur de la RFA auprès du Saint-Siège écrivit à Bonn que «Casaroli paraît disposé à aller au-delà de la position actuelle officielle du Saint-Siège; il est résolu à garantir la présence vaticane à la Conférence $»^{21}$. Cette position ne faisait pas l'unanimité et l'ambassadeur indiquait que, sur la question du nouveau rôle du Saint-Siège dans les relations internationales, d'autres «personnalités en Vatican, en particulier l'archevêque Benelli [numéro deux de la Secrétairerie d'État] et également le secrétaire d'État Villot, paraissent être beaucoup plus prudentes $»^{22}$.

20. Position développée par A. Casaroli dans Nella Chiesa per il mondo, Milan, Rusconi, 1987, spéc. p. 414-416.

21. Rapport du 28 mars 1972 de Rome à Bonn, PAAA, B28 109300; d'après M. Faggioli, op. cit., p. 192, note 44.

22. «Andere Persönlichkeiten im Vatikan, insbesondere Erzbischof Benelli und wohl auch Kardinalstaatssekretär Villot, dürften in dieser Frage wesentlich behutsamer und zurückhaltender sein », ibid. Le mécontentement de Mgr Benelli se retrouve aussi dans la dépêche du 25 juillet 1972 remise à Bonn par l'ambassadeur de la RFA, qui commentait la visite de l'ambassadeur finlandais auprès de Paul VI, in PAAA B28 109300 (d'après M. Faggioli, op. cit., p. 193, note 45). Pour les différences d'attitude entre Mgr Benelli et Mgr Casaroli, et l'augmentation progressive des charges confiées par le pape à Casaroli, voir la relation de l'ambassade allemande sur la réforme de la Curie romaine, 10 octobre 1968, in PAAA, B 26 373; cité par M. Faggioli, «Ostpolitik vaticana e la "questione tedesca", 1958-1968», Contemporane n 3, juillet 2007, p. 415, note 54. 
C'est d'ailleurs comme on le verra Mgr Casaroli et non le cardinal Villot qui sera le signataire de l'Acte final d'Helsinki ${ }^{23}$.

Après une petite hésitation d'ordre juridique, le Saint-Siège se prononça de manière très positive pour la concrétisation du projet: selon Mgr Silvestrini, «la participation à la conférence d'Helsinki a été une décision prise par Paul VI avec clairvoyance et courage. C'était la première fois que le Saint-Siège participait à part entière à une conférence de caractère politique. Le Pacte de Varsovie l'avait invité, évidemment pour obtenir un aval moral; beaucoup craignaient que cela puisse déboucher sur un appui donné à la politique soviétique en Europe. Le Saint-Siège a accepté ce risque pas seulement pour les catholiques, mais pour tous les croyants, même pour tous les hommes, conformément à la déclaration Dignitatis Humanae du Vatican II sur la liberté religieuse $»^{24}$.

Le 22 juillet 1973 le pape Paul VI annonça la participation du Saint-Siège aux négociations de la CSCE et désigna comme son représentant diplomatique le secrétaire du Conseil pour les affaires publiques de l'Église, l'archevêque Agostino Casaroli ${ }^{25}$. Des négociations longues et fatigantes avec les représentants des gouvernements socialistes furent engagées dans le but d'obtenir une sorte de reconnaissance politique, qui permette ensuite des progrès en matière de liberté religieuse. Elles aboutirent à une légitimation contenue dans le septième principe de l'Acte final, considéré par l'Église catholique comme conforme à ses exigences ${ }^{26}$. Au cours de la pré-consultation du 6 mars 1973, le prononce à Helsinki,

23. Antoine Wenger, Le cardinal Jean Villot (1905-1979) secrétaire d'État de trois papes, Paris, Desclée de Brouwer 1989, p. 158.

24. A. Silvestrini, «L'Ostpolitik de Paul VI», in Paul VI et la vie internationale, actes des journées d'études d'Aix-en-Provence, 18 et 19 mai 1990, Istituto Paolo VI di Brescia (éd.), Rome, Studium 1992, p. 113-129.

25. "Il discorso dell'onomastico" nell'udienza al sacro collegio. Un balzo in avanti sulla linea del Concilio, L'Osservatore Romano du 22-23 juillet 1973, p. 2.

26. Mgr Casaroli remit le 20 avril 1973 un aide-mémoire (Consilium pro publicis ecclesiae negotiis n. 2575/73) à l' ambassadeur de France auprès du SaintSiège, qui le transmit au ministre des Affaires étrangères, Michel Jobert (doc. n. 118/EU). Le passage relatif à la Coopération dans le champ de la culture et des contacts entre les hommes précise que la commission inclura parmi les mesures à adopter l'accroissement des échanges d'informations religieuses et des possibilités de contacts et de rencontres entre les personnes et entre les organisations confessionnelles pour des motifs religieux. Sur ces questions, voir G. Barberini, Pagine di storia contemporanea: La Santa Sede alla Conferenza di Helsinki, Sienne, Cantagalli, 2010. 
Mgr Zabkar, dit du rendez-vous d'Helsinki qu'il était une «grande chance» pour les peuples de l'Europe, en mettant en évidence que pour le Saint-Siège la question de la protection et du développement des droits de l'homme était importante: «Par sa tâche spirituelle et morale au service de l'Homme, la plus grande attention du SaintSiège va à la protection et au renforcement, sans exception, des droits de l'homme. Dans ce contexte, il est naturel qui revienne au Saint-Siège la possibilité de prononcer un mot particulier sur la liberté religieuse $»^{27}$.

La présence du Saint-Siège à la Conférence pour la sécurité et la coopération en Europe (CSCE) - qui pendant quelques années, attira l'intérêt et l'attention de l'opinion publique, surtout en Europe - permit à Mgr Casaroli d'établir des relations utiles avec les responsables de la politique étrangère des États socialistes: "C'est précisément à Helsinki, à l'occasion de la première phase de la Conférence, en juillet 1973, que j'eus un rendez-vous avec le ministre des affaires étrangères polonais Stefan Olszowski», a expliqué Mgr Casaroli ${ }^{28}$. L'objet du rendez-vous naturellement, comme toutes les autres rencontres tenues ces jours-là dans la capitale finlandaise, ce furent les problèmes liés à la sécurité et la coopération en Europe, surtout la relation entre la Pologne et le Saint-Siège et la reprise des contacts suspendus en novembre deux années auparavant. Ces relations avec les responsables de la politique étrangère des États socialistes ont - mais de manière partielle - favorisé l'évolution des relations bilatérales entre le Saint-Siège et chacun des États socialistes, et la redéfinition de son rôle international. Les contributions politiques et diplomatiques du Saint-Siège à la conférence d'Helsinki ont permis l'assouplissement des normes coercitives contre les catholiques. Un procès-verbal de l'entrevue de février 1974 entre Casaroli et Olszowski témoigne de cette évolution: «Le problème de la sécurité européenne préoccupe le Saint-Siège de manière profonde. La participation du Saint-Siège à la Conférence a suscité surprise et merveille. Le Saint-Siège d'habitude agit, mais sans prendre part comme il a fait pour cette Conférence $»^{29}$.

27. Cité par la Documentation catholique, $\mathrm{n}^{\circ}$ 1630, 15 avril 1973.

28. A. Casaroli, Il martirio della pazienza..., op. cit., p. 308.

29. Verbale dei colloqui di Mons. Casaroli con il Sig. S. Olszowski, Ministro degli Esteri della Polonia, a cura di Mons. Montalvo <Incontri del 4 e 5 febbraio 
Au sujet des problèmes liés à la sécurité et à la coopération en Europe, Mgr Casaroli dans son intervention à la séance plénière du 6 juillet 1973 affirma: "Le Saint-Siège estime, que, pour atteindre réellement les objectifs visés par la Conférence dans le domaine de la sécurité, il est d'un intérêt capital de trouver un accord commun, conformément au paragraphe 21 des "Recommandations finales des consultations de Helsinki" ${ }^{30}$, sur des mesures concrètes capables de "rendre effectif le non-recours à la menace ou à l'emploi de la force", et, entre autres, "une méthode de règlement pacifique des différends entre États participants"[...]. En effet, le Saint-Siège attribue une valeur fondamentale aux conditions objectives de la paix et à la volonté politique des États quant à l'observance des principes régissant leur coexistence pacifique et leur collaboration mutuelle et fructueuse: dans le respect de la souveraineté des Étatsmembres, bien sûr, mais aussi dans la recherche efficace d'une solution ou d'un compromis sauvegardant l'intérêt commun de la sécurité $»^{31}$.

\section{L'Église de Paul VI et «L'esprit d’Helsinki»}

Le $1^{\text {er }}$ août 1975 Mgr Casaroli signa l'Acte final d'Helsinki relatif à la Conférence pour la sécurité et la coopération en Europe; ce document, suivant les bonnes résolutions des signataires, devait mettre un terme à la longue phase de méfiance et de renfermement mutuels entre les États. Dans un entretien au quotidien italien Paese-sera, Mgr Casaroli reconnaît que la conférence d'Helsinki ne va pas résoudre les problèmes des rapports entre l'Église et les États socialistes. Mais il ajoute «que la création d'une atmosphère de confiance entre tous les États de l'Europe est un bénéfice aussi pour l'Église [...]. À cet égard également les contacts établis lors des travaux d'Helsinki ont été utiles ${ }^{32}$. Les gouvernements des

$\overline{\text { 1974. Montalvo }}>$ del 4 febbraio 1974, in A.S.Pa., Fondo Casaroli, b. 3 «Polonia», f. 41 , s.f. 1 .

30. Dont on peut consulter le texte sur le site de l'OSCE: www.osce.org/fr/ $\mathrm{mc} / 40214$

31. "Le Saint-Siège et la sécurité européenne. Intervention de Mgr Casaroli à la Conférence d'Helsinki au cours de la séance plénière du 6 juillet 1973 », la Documentation catholique $\mathrm{n}^{\circ}$ 1637, 5-19 août 1973, p. 723.

32. Interview de Mgr Agostino Casaroli, Paese-sera, 8 juillet 1973. 
pays communistes lui envoyèrent des invitations officielles qu'il fut «autorisé à accepter: Pologne (février 1974, trois mois après l'audience donnée par le pape au Ministre des affaires étrangères polonais Stefan Olszowski), Tchécoslovaquie (février 1975), Allemagne de l'Est (juin 1975), Bulgarie (novembre 1976) $»^{33}$.

«L'esprit d'Helsinki» produisit quelque changement dans les pays de l'Europe de l'Est: «Liée au nouveau climat créé par la Conférence a été l'audience pontificale du Président bulgare, Todor Zhivkov. Fruit visible du rendez-vous a été la nomination de deux évêques, Mgr Dobranov et Mgr Séirécov, et l'invitation de Casaroli par le ministre des Affaires étrangères Mladenov pour une visite officielle en Bulgarie $»^{34}$. En Hongrie, les conditions pour l'Église furent améliorées, mais une partie de l'épiscopat local continua de subir l'influence du gouvernement de Budapest. En Tchécoslovaquie la diplomatie du Saint-Siège se heurta à la dure opposition du gouvernement de Prague: la «politique du dialogue» fut en pratique un échec. Le constat vaut également pour les catholiques, ukrainiens et roumains, qui en plus de l'opposition des régimes politiques devaient affronter celle des églises orthodoxes. Le pape Paul VI maintint la politique du dialogue, «un dialogue franc et ouvert, qu'on ne peut considérer sans résultats $»^{35}$; toutefois, «alors qu'il louait le travail de ses représentants, il ne pouvait pas cacher avoir beaucoup souffert à cause de l'attitude hostile et finalement négative des représentants des États. $»^{36}$

La figure d'Agostino Casaroli reste comme celle d'un artisan de paix, animé de convictions profondes. «L'esprit d'Helsinki», dans sa mise en œuvre, rencontra certes de nombreux obstacles; mais il demeure un modèle pour les catholiques engagés dans la défense des droits civiques, parce qu'il lie la cause de leur Église à celle de la paix : pour Mgr Casaroli, c'était à cette condition, par la recherche permanente du dialogue, que l'Église avait une chance d'accomplir sa mission historique, en Europe et dans le monde.

m.lavopa@libero.it

33. Voir A. Casaroli, Il martirio della pazienza..., op. cit., p. 115-6.

34. Ibid., p. 322-3.

35. Discours du pape Paul VI au Sacré Collège, 21 juin 1976, in Insegnamenti di Paolo VI, t. XVI, p. 498-507.

36. A. Casaroli, op. cit., p. 172. 